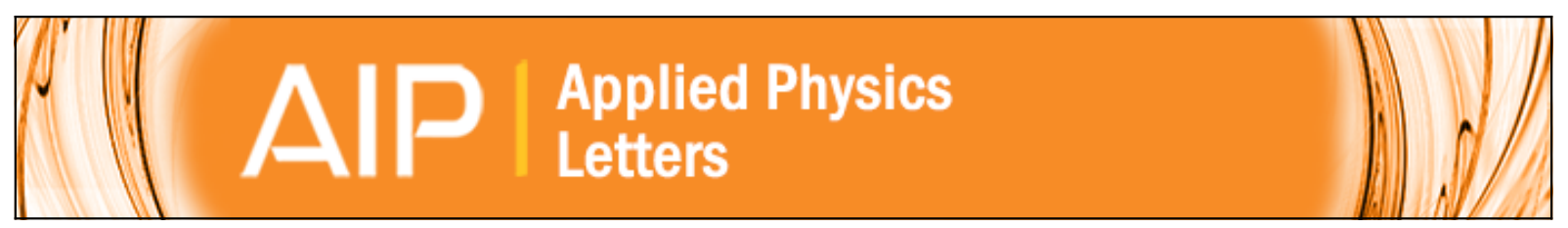

\title{
Antisites and anisotropic diffusion in GaAs and GaSb
}

H. A. Tahini, A. Chroneos, H. Bracht, S. T. Murphy, R. W. Grimes, and U. Schwingenschlögl

Citation: Applied Physics Letters 103, 142107 (2013); doi: 10.1063/1.4824126

View online: http://dx.doi.org/10.1063/1.4824126

View Table of Contents: http://scitation.aip.org/content/aip/journal/apl/103/14?ver=pdfcov

Published by the AIP Publishing

\section{NEW}

Model PS-100

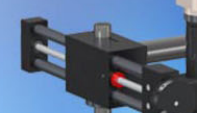

Preconfigured Tabletop

Probe Station

\section{Lake Shore CRYOTRONICS}

An affordable solution for a wide range of research 


\title{
Antisites and anisotropic diffusion in GaAs and GaSb
}

\author{
H. A. Tahini, ${ }^{1, a)}$ A. Chroneos, ${ }^{2, b)}$ H. Bracht, ${ }^{3, c)}$ S. T. Murphy, ${ }^{1}$ R. W. Grimes, ${ }^{1, d)}$ \\ and U. Schwingenschlögl ${ }^{4, e)}$ \\ ${ }_{1}^{1}$ Department of Materials, Imperial College London, London SW7 2AZ, United Kingdom \\ ${ }^{2}$ Engineering and Innovation, The Open University, Milton Keynes MK7 6AA, United Kingdom \\ ${ }^{3}$ Institute of Materials Physics, University of Münster, Wilhelm-Klemm-Strasse 10, D-48149 Münster, \\ Germany \\ ${ }^{4}$ PSE Division, KAUST, Thuwal 23955-6900, Saudi Arabia
}

(Received 29 July 2013; accepted 19 September 2013; published online 2 October 2013)

\begin{abstract}
The significant diffusion of Ga under Ga-rich conditions in GaAs and GaSb is counter intuitive as the concentration of $\mathrm{Ga}$ vacancies should be depressed although $\mathrm{Ga}$ vacancies are necessary to interpret the experimental evidence for Ga transport. To reconcile the existence of Ga vacancies under Ga-rich conditions, transformation reactions have been proposed. Here, density functional theory is employed to calculate the formation energies of vacancies on both sublattices and the migration energy barriers to overcome the formation of the vacancy-antisite defect. Transformation reactions enhance the vacancy concentration in both materials and migration energy barriers indicate that Ga vacancies will dominate. (C) 2013 AIP Publishing LLC. [http://dx.doi.org/10.1063/1.4824126]
\end{abstract}

A number of binary III-V compound semiconductors have high electron mobility, narrow direct band gap and can be lattice matched with ternary or quaternary III-V compounds. $^{1-5}$ These properties make them technologically important for microelectronic applications, diodes, photovoltaics, and solar cells. ${ }^{1}$ For example, GaAs is being considered for advanced nanoelectronic devices as the recent trend is to replace silicon $(\mathrm{Si})$ in devices with substrates that exhibit higher electron mobility. ${ }^{6,7}$ Interestingly, theoretical studies can provide a fundamental insight in the defect processes of group III-V compounds (such as growth in GaAs). ${ }^{5}$

Interestingly, Bracht et al. ${ }^{8,9}$ have determined that selfdiffusion in $\mathrm{GaSb}$ is asymmetric with $\mathrm{Ga}$ diffusing more rapidly than $\mathrm{Sb}$ by over three orders of magnitude near the melting temperature. Furthermore, Ga self-diffusion is more significant than $\mathrm{Sb}$ even under Ga-rich conditions. Gadiffusion is mainly vacancy-mediated in GaSb so one needs to understand the large disparity between the diffusion coefficients of $\mathrm{Ga}$ and $\mathrm{Sb}$ and the role of $\mathrm{Ga}$ vacancies $\left(V_{\mathrm{Ga}}\right)$ even under Ga-rich conditions.

Bracht and Brotzmann ${ }^{6}$ explain the diffusion of $\mathrm{Zn}$ in GaAs via the kick-out mechanism that involves neutral and single positively charged self-interstitials. In addition, $V_{\mathrm{Ga}}$ contributes to $\mathrm{Zn}$ diffusion via the dissociative mechanism. ${ }^{6} \mathrm{Zn}$ diffusion in GaAs is effectively a probe to determine the individual contributions of charged $\mathrm{Ga}$ interstitials $\left(\mathrm{Ga}_{i}\right)$ and $V_{\mathrm{Ga}}$ to the total $\mathrm{Ga}$ self-diffusion coefficient. For $\mathrm{Zn}$ diffusion under As-rich conditions, Bracht and Brotzmann ${ }^{6}$ fitted the experimental $\mathrm{Zn}$ profiles data for the individual contribution of $\mathrm{Ga}_{i}$ and $V_{\mathrm{Ga}}$ to Ga self-diffusion in GaAs, which are consistent with the total self-diffusion coefficient. That is, the individual contributions are lower than the total Ga self-diffusion. However, the situation is very different under Ga-rich conditions. Although

\footnotetext{
a)Electronic mail: h.tahini@imperial.ac.uk

b)alex.chroneos@open.ac.uk

c)bracht@uni-muenster.de

d)r.grimes@imperial.ac.uk

e)Udo.Schwingenschlogl@kaust.edu.sa
}

the same diffusion mechanisms (see Eqs. (2)-(4) of Ref. 6) describe the experimental $\mathrm{Zn}$ profiles and even the Ga profiles, the individual contributions of neutral and positive charged $V_{\mathrm{Ga}}$ reduced to standard conditions (electronically intrinsic and to an As pressure of $1 \mathrm{~atm}$ ), exceed the total Ga self-diffusion coefficient. ${ }^{10}$ It is striking that the same model that works for As-rich conditions also accurately reproduces $\mathrm{Zn}$ profiles obtained under Ga-rich conditions. One counter intuitive idea that may nevertheless explain the situation is that significant concentrations of $V_{\mathrm{Ga}}$ exist and can evolve from $V_{\mathrm{As}}$ even under Ga-rich conditions.

Apart from the investigation of vacancies, the present study will also focus on the associations of antisites defects with vacancies. Using density functional theory (DFT), we present a detailed investigation of the point defects under different compositional conditions (stoichiometric, Ga-rich, and As/Sb-rich) and Fermi levels. To gain a complete understanding of the processes, we also consider the kinetics of the transformation reactions.

The results were generated using density functional theory as implemented in the VASP code. ${ }^{11}$ Exchange and correlation were described according to the formulation of Perdew et al. ${ }^{12}$ Pseudopotentials were generated using the projector-augmented method ${ }^{13}$ in which the 3 electrons of the group III atoms and 5 electrons of the group IV atoms were treated as valence. Wavefunctions were expanded to a kinetic energy cut-off of $400 \mathrm{eV}$.

The calculations were carried out in 216 atom supercells. Integration over the Brillioun zone was performed using a $2 \times 2 \times 2$ grid generated according to Monkhorst and Pack. ${ }^{14}$ Energies and forces were iterated up to tolerances of $1 \times 10^{-5} \mathrm{eV}$ and $1 \times 10^{-2} \mathrm{eV} / \AA$, respectively. Defect formation energies were calculated according to

$$
E^{f}=E_{\mathrm{tot}}(D, q)-E_{\mathrm{tot}}(\text { perfect })+\sum_{\alpha} n_{\alpha} \mu_{\alpha}+q \mu_{\mathrm{e}}+E_{\text {corr }}
$$

where $E_{\mathrm{tot}}(D, q)$ and $E_{\mathrm{tot}}$ (perfect) are the total energies of the defective and perfect cells, respectively. $n_{\alpha}$ represents the 
number of atoms added or removed and $\mu_{\alpha}$ is the corresponding chemical potential. Charged defect-defect interactions were accounted for using the recent technique due to Freysoldt et al. ${ }^{15}$ which has been applied in several previous studies and appears in the equation above as $E_{\text {corr }}$. To calculate the migration barriers, we employed the nudged elastic band technique with the climbing image functionality (CI-NEB). ${ }^{16}$

It is important to consider how $V_{\mathrm{Ga}}$ is relevant in GaAs and GaSb. When III-V compounds are stoichiometric, they are ordered binary compounds with the zinc-blende structure ( $F \overline{4} 3 m$, space group No. 216). The zinc-blende structure has two sublattices, with each sublattice being occupied, ideally, by atoms of one kind. The four nearest neighbor sites of every lattice site lie on the other sublattice, whereas the second neighbour sites lie on the same sublattice. In the plane-passing mechanism proposed by Bockstedte and Scheffler ${ }^{17}$ (for Ga diffusion in GaAs), a Ga atom at a second nearest neighbor position with respect to a $V_{\mathrm{Ga}}$ moves towards it leaving its own site vacant. Thus, the Ga atom moves towards the interstitial region along the diffusion plane that is perpendicular to the (110) plane (see also Fig. 3 in Ref. 17). The advantage of the plane-passing mechanism is that it does not necessitate the formation of defect complexes (such as $V_{\mathrm{Sb}} V_{\mathrm{Ga}}$ in the triple-defect mechanism ${ }^{18}$ ) or the creation of antisite disorder (such as in the ten-jump process ${ }^{19}$ ). This is because Ga self-diffusion is taking place on the Ga-sublattice and As self-diffusion on the As sublattice. In recent DFT studies, El-Mellouhi and Mousseau ${ }^{20,21}$ considered other possible mechanisms for Ga self-diffusion in GaAs but predicted that the plane-passing mechanism is the most energetically favorable for $V_{\mathrm{Ga}}^{0}$ and $V_{\mathrm{Ga}}^{1-}$ (both with diffusion barriers of $1.7 \mathrm{eV}$ ) but also for $V_{\mathrm{Ga}}^{2-}$ (diffusion barrier $1.85 \mathrm{eV}$ ).

Results presented in Fig. 1(a) show that in GaSb, irrespective of the composition conditions (i.e., $\mathrm{Ga}$ or $\mathrm{Sb}$ rich) or Fermi level, the isolated $V_{\mathrm{Ga}}$ defect has a lower formation energy than the antimony vacancy $\left(V_{\mathrm{Sb}}\right)$. Fig. 1(b) shows that for GaAs, the $V_{\mathrm{As}}$ is more favorable than the $V_{\mathrm{Ga}}$ only for some $p$-type conditions in stoichiometric and Ga-rich compositions, although, it is always most favorable in As-rich compositions.

Bracht et al. ${ }^{8}$ have proposed the transformation reaction $V_{\mathrm{As}} \rightarrow V_{\mathrm{Ga}}+\mathrm{Ga}_{\mathrm{As}}$ as a way to produce $V_{\mathrm{Ga}}$ from $V_{\mathrm{As}}$ in GaAs under Ga-rich conditions. In essence, Bracht et al. ${ }^{8}$ considered the formation of two isolated species, however, as an intermediate process the vacancy-antisite pair is formed. Formally, the dissociation energy required to break up the pair defect also needs to be calculated to gain a full understanding of the defect processes. This will involve the $V_{\mathrm{Ga}}$ migrating away from the pair via the plane-passing mechanism, ${ }^{17}$ effectively with a Ga atom at a second nearest neighbor position (with respect to a $V_{\mathrm{Ga}}$ ) moving towards it, leaving its own site vacant. Here, we assume that under experimental conditions, all the vacancies will contribute to diffusion at their sublattice. For such conditions, our calculations, illustrated in Fig. 1(b), reveal that it is energetically favourable to form isolated vacancies. In addition, we calculated the transformation reaction to form antisite-vacancy pairs for both materials to assess the impact on the $V_{\mathrm{Ga}}$ concentration, as was proposed experimentally. Fig. 1 reports the antisite-vacancy pair formation energies for the most stable charge states as a function of the Fermi level for stoichiometric, III-rich, and V-rich conditions in both $\mathrm{GaSb}$ and GaAs. The most stable cluster configuration is predominantly the nearest neighbour $\mathrm{Ga}_{\mathrm{As}} V_{\mathrm{Ga}}$ in GaAs and $\mathrm{Sb}_{\mathrm{Ga}} V_{\mathrm{Sb}}$ in $\mathrm{GaSb}$ regardless of composition. Considering first $\mathrm{GaSb}$ under Ga-rich conditions, the $\mathrm{Ga}_{\mathrm{Sb}} V_{\mathrm{Ga}}$ cluster has a low formation energy under $n$-type conditions supporting the transformation reaction $V_{\mathrm{Sb}} \rightarrow V_{\mathrm{Ga}}+\mathrm{Ga}_{\mathrm{Sb}}$ that was previously proposed to explain the $V_{\mathrm{Ga}}$ mediated diffusion of $\mathrm{Ga}$ in

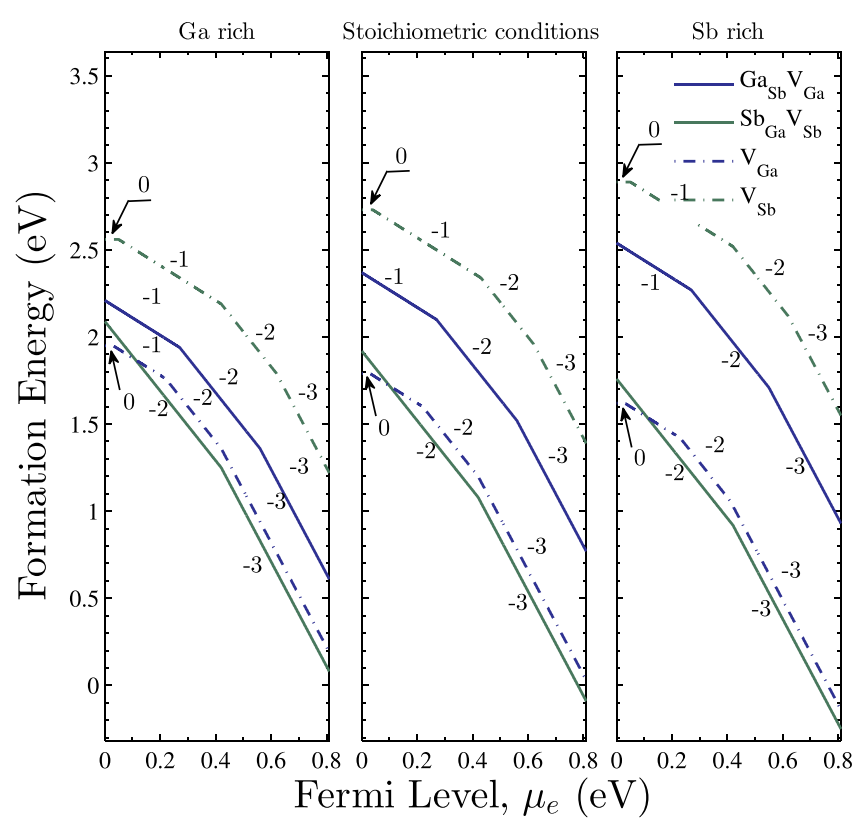

(a)

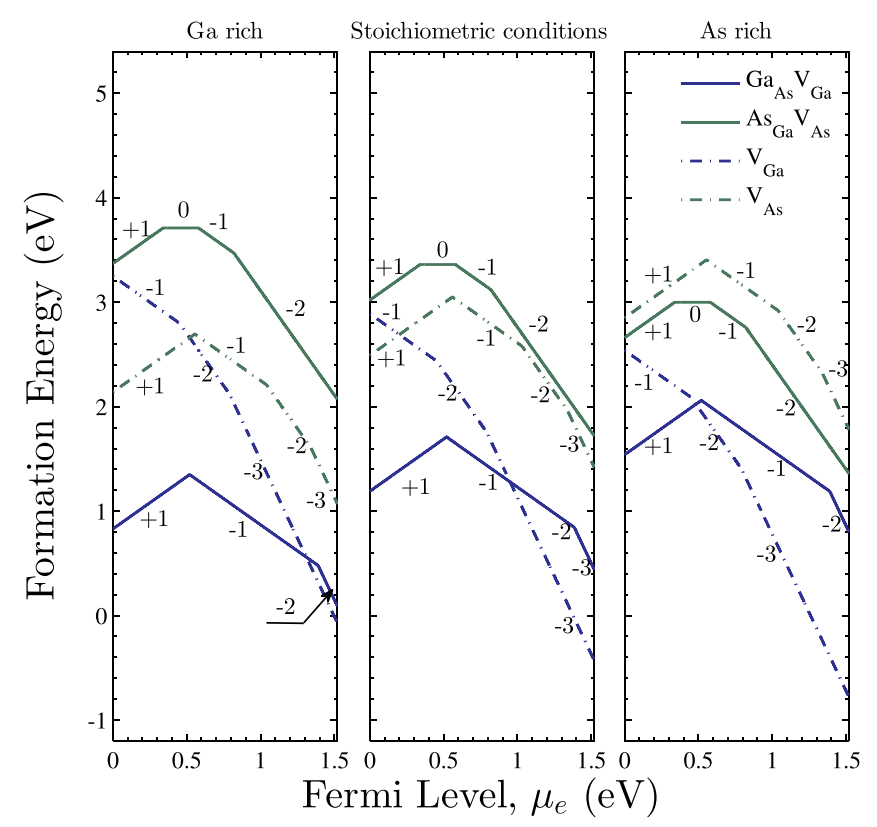

(b)

FIG. 1. Lowest energy vacancy and antisite-vacancy pair formation energies assuming the most stable charge state as a function of the Fermi level for stoichiometric, III-rich and V-rich conditions for (a) GaSb and (b) GaAs. Numbers in the figures represent the charge state of the respective defects. 


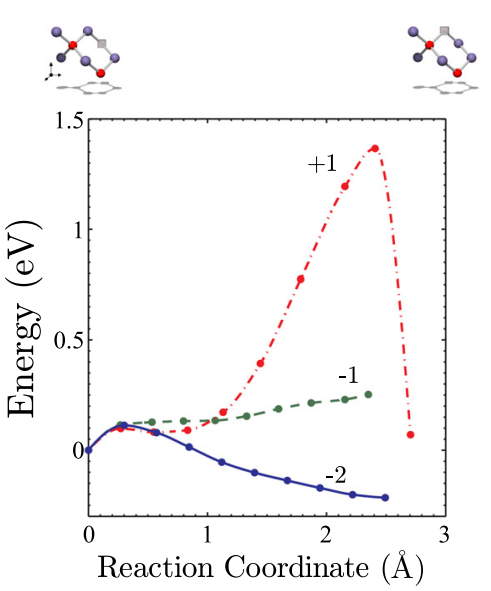

(a)

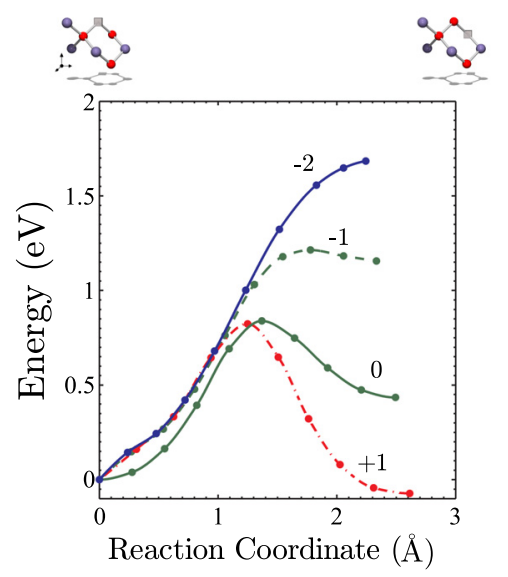

(b)
FIG. 2. The migration energy barriers for (a) $V_{\mathrm{As}} \rightarrow$ $V_{\mathrm{Ga}}+\mathrm{Ga}_{\mathrm{As}}$ and (b) $V_{\mathrm{Ga}} \rightarrow V_{\mathrm{As}}+\mathrm{As}_{\mathrm{Ga}}$ transformation reactions in GaAs. On the top of the figure is the initial and final state of the transformation reaction. Cubes represent the vacant site, red spheres the As atoms and purple spheres the Ga atoms. The reaction coordinates represent the distance between the images along the path of the diffusing species. Numbers in the figures represent the charge state of the respective defects.
GaSb under Ga-rich conditions. ${ }^{8}$ The present study, however, also supports the view that isolated $V_{\mathrm{Ga}}$ is produced even under Ga-rich conditions and is prevalent over $V_{\mathrm{Sb}}$ (Fig. 1(a)). Again, this is consistent with the experimental evidence of significantly higher Ga self-diffusion compared with $\mathrm{Sb}$ self-diffusion in GaSb. At any rate, the transformation reaction can add complementary $V_{\mathrm{Ga}}$ to the system.

In $\mathrm{GaAs}$ under Ga-rich conditions, $\mathrm{Ga}_{\mathrm{As}} V_{\mathrm{Ga}}$ has low formation energies especially for low Fermi levels where $V_{\text {As }}$ have lower formation energies than $V_{\mathrm{Ga}}$ (see Fig. 1(b)). Accordingly, we expect through the transformation reaction that additional $V_{\mathrm{Ga}}$ is added to the system. Therefore, GaAs and $\mathrm{GaSb}$ are similar under Ga-rich conditions. Where the two materials behave differently is that under all conditions the $\mathrm{Sb}_{\mathrm{Ga}} V_{\mathrm{Sb}}$ pair exhibits a lower formation energy than the $V_{\mathrm{Ga}}$, the analogous pair in GaAs (i.e., the $\mathrm{As}_{\mathrm{Ga}} V_{\mathrm{As}}$ pair) exhibits significantly higher formation energy under $\mathrm{Ga}$ rich conditions. The lower formation energies of the $\mathrm{Sb}_{\mathrm{Ga}} V_{\mathrm{Sb}}$ pair suggest that the transformation reaction $V_{\mathrm{Ga}} \rightarrow V_{\mathrm{Sb}}+\mathrm{Sb}_{\mathrm{Ga}}$ would form $V_{\mathrm{Sb}}$ even under Sb-rich conditions, something that was not observed experimentally. ${ }^{8}$ Hence to understand the absence of these thermodynamically favourable species, the kinetics of the transformation reactions are considered next by calculating the migration energies for these processes.

While the formation of $V_{\mathrm{Ga}}$ via transformation reactions may increase the $V_{\mathrm{Ga}}$ concentration one has to consider the migration energy barrier that must be overcome to form the defect pair. A schematic representation of the transformation reaction $V_{\mathrm{As}} \rightarrow V_{\mathrm{Ga}}+\mathrm{Ga}_{\mathrm{As}}$ in GaAs is provided at the top of Fig. 2(a). In essence, a nearest neighbour $\mathrm{Ga}$ atom moves into the vacant As site. This leads to the formation of the Ga antisite and a vacant $\mathrm{Ga}$ site. Analogous mechanisms were also considered for $V_{\mathrm{Ga}} \rightarrow V_{\mathrm{As}}+\mathrm{As}_{\mathrm{Ga}}, V_{\mathrm{Sb}} \rightarrow V_{\mathrm{Ga}}+\mathrm{Ga}_{\mathrm{Sb}}$, and $V_{\mathrm{Ga}} \rightarrow V_{\mathrm{Sb}}+\mathrm{Sb}_{\mathrm{Ga}}$ in Figs. 2(b), 3(a), and 3(b).

Figs. 2 and 3 report the energies along the path defined on the top of the figures for GaAs and $\mathrm{GaSb}$, respectively. The charge states considered correspond to the dominant values of the vacancy-antisite pair identified in Fig. 1. It can be observed by comparing Figs. 2(a) and 2(b) and Figs. 3(a) and 3(b) that the lowest energy barriers, irrespective of the charge states, are for the production of $V_{\mathrm{Ga}}$ via the respective transformation reactions. For example, considering GaAs (see Fig. 2), the barrier to form doubly negatively charged $V_{\mathrm{As}}+\mathrm{As}_{\mathrm{Ga}}$ pairs (via $V_{\mathrm{Ga}} \rightarrow V_{\mathrm{As}}+\mathrm{As}_{\mathrm{Ga}}$, Fig. 2(a)) is more than $1.3 \mathrm{eV}$ higher compared with the barrier to form the doubly negatively charged $V_{\mathrm{Ga}}+\mathrm{Ga}_{\mathrm{As}}$ pairs (via $V_{\mathrm{As}} \rightarrow V_{\mathrm{Ga}}$ $+\mathrm{Ga}_{\mathrm{As}}$, Fig. 2(b)).

It is evident that the transformation reactions leading to the production of $V_{\mathrm{Ga}}$ are energetically favourable over the analogous reactions for all conditions in both materials (Figs. 2 and 3). For GaSb this supports the model proposed by Bracht et al., ${ }^{8}$ namely, that under Ga-rich conditions transformation reactions $\left(V_{\mathrm{Sb}} \rightarrow V_{\mathrm{Ga}}+\mathrm{Ga}_{\mathrm{Sb}}\right.$ ) lead to the formation of Ga vacancies (Fig. 3). At high Fermi levels, the energy of this

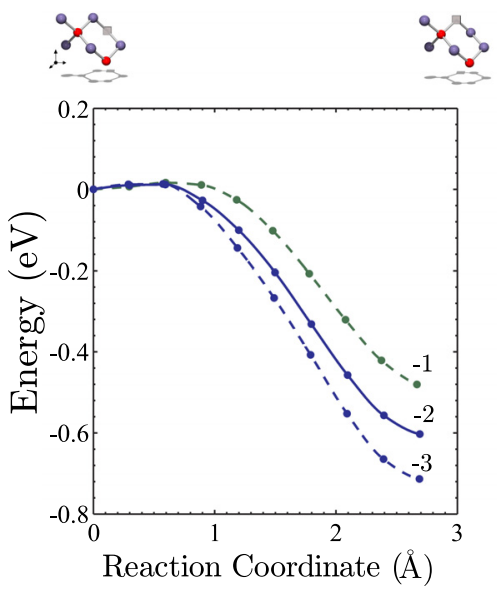

(a)

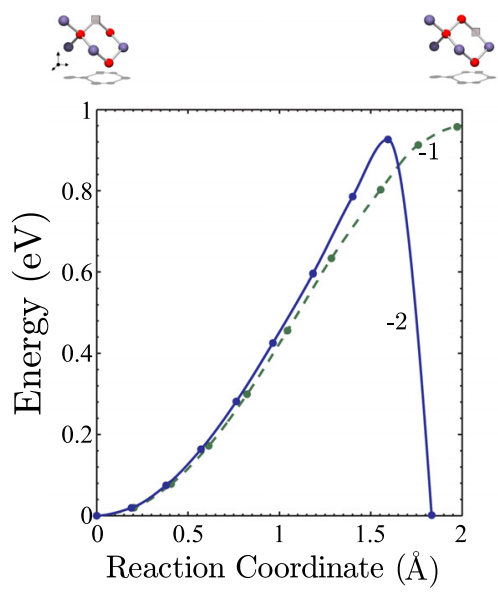

(b)
FIG. 3. The migration energy barriers for (a) $V_{\mathrm{Sb}} \rightarrow$ $V_{\mathrm{Ga}}+\mathrm{Ga}_{\mathrm{Sb}}$ and (b) $V_{\mathrm{Ga}} \rightarrow V_{\mathrm{Sb}}+\mathrm{Sb}_{\mathrm{Ga}}$ transformation reactions in GaSb. On the top of the figure is the initial and final state of the transformation reaction. Cubes represent the vacant site, red spheres the $\mathrm{Sb}$ atoms and purple spheres the $\mathrm{Ga}$ atoms. The reaction coordinates represent the distance between the images along the path of the diffusing species. Numbers in the figures represent the charge state of the respective defects. 
process is very low leading to the transformation of $V_{\mathrm{Sb}}$ to additional $V_{\mathrm{Ga}}$. Conversely, the process $V_{\mathrm{Ga}} \rightarrow V_{\mathrm{Sb}}+\mathrm{Sb}_{\mathrm{Ga}}$ in $\mathrm{GaSb}$ (which appears favourable at high Fermi energies in terms of formation energies in Fig. 1(a)) is hindered by the high migration energy barriers (refer to Fig. 3(b)). The picture in GaAs is very similar, that is, the migration energy barriers for the production of $V_{\mathrm{Ga}}$ via the transformation reactions are lower as compared with the production of the $V_{\mathrm{As}}$.

The results of the present study lead to counterintuitive conclusions as it is predicted that the concentration of $V_{\mathrm{Ga}}$ or $\mathrm{Ga}_{\mathrm{As}} V_{\mathrm{Ga}}$ pairs is significant and dominant over group- $\mathrm{V}$ vacancies even under Ga-rich conditions for both $\mathrm{GaSb}$ and GaAs. They are consistent though with the experimentally observed diffusion behavior in both materials. Transformation reactions under Ga-rich conditions can provide complementary $V_{\mathrm{Ga}}$ but these reactions are not necessary to explain the existence of $V_{\mathrm{Ga}}$ as they exhibit formation energies that are compatible with high Ga diffusion. The kinetics of the processes are, however, necessary to explain the suppression of $V_{\mathrm{Sb}}$ as the migration energies of the transformation reaction establish the dominance of the $V_{\mathrm{Ga}}$ over $V_{\mathrm{As}}$ or $V_{\mathrm{Sb}}$ for $\mathrm{GaAs}$ and $\mathrm{GaSb}$.

This publication was based on research partially supported by King Abdullah University for Science and Technology (KAUST). Computing resources were provided by the Shaheen supercomputer at KAUST and the High Performance Computing (HPC) facility of Imperial College London.

${ }^{1}$ P. S. Dutta, H. L. Bhat, and V. Kumar, J. Appl. Phys. 81, 5821 (1997).

${ }^{2}$ J. A. del Alamo, Nature (London) 479, 317 (2011).
${ }^{3}$ L. Lin and J. Robertson, Appl. Phys. Lett. 98, 082903 (2011); H. A. Tahini, A. Chroneos, S. T. Murphy, U. Schwingenschlögl, and R. W. Grimes, J. Appl. Phys. 114, 063517 (2013); A. Chroneos and H. Bracht, J. Appl. Phys. 104, 093714 (2008); S. T. Murphy, A. Chroneos, R. W. Grimes, C. Jiang, and U. Schwingenschlögl, Phys. Rev. B 84, 184108 (2011).

${ }^{4}$ H. P. Komsa and A. Pasquarello, J. Phys.: Condens. Matter 24, 045801 (2012); D. Colleoni and A. Pasquarello, Microelectron. Eng. 109, 50 (2013).

${ }^{5}$ A. Kley, P. Ruggerone, and M. Scheffler, Phys. Rev. Lett. 79, 5278 (1997); B. A. Joyce and D. D. Vvedensky, Mater. Sci. Eng. R 46, 127 (2004); J. L. Roehl, A. Kolagatla, V. K. K. Gangur, S. V. Khare, and R. J. Phaneuf, Phys. Rev. B 82, 165335 (2010); S. T. Murphy, A. Chroneos, C. Jiang, U. Schwingenschlögl, and R. W. Grimes, Phys. Rev. B 82, 073201 (2010); J. L. Rohl, S. Aravelli, S. V. Khare, and R. J. Phaneuf, Surf. Sci. 606, 1303 (2012).

${ }^{6}$ H. Bracht and S. Brotzmann, Phys. Rev. B 71, 115216 (2005).

${ }^{7}$ W. S. Jung, J. H. Park, A. Nainani, D. Nam, and K. C. Saraswat, Appl. Phys. Lett. 101, 072104 (2012).

${ }^{8}$ H. Bracht, S. P. Nicols, W. Walukiewicz, J. P. Silveira, F. Briones, and E. E. Haller, Nature (London) 408, 69 (2000).

${ }^{9}$ H. Bracht, S. P. Nicols, E. E. Haller, J. P. Silveira, and F. Briones, J. Appl. Phys. 89, 5393 (2001).

${ }^{10}$ H. Bracht, M. S. Norseng, E. E. Haller, and K. Eberl, Physica B 309-310, 831 (2001).

${ }^{11}$ G. Kresse and J. Furthmüller, Phys. Rev. B 54, 11169 (1996).

${ }^{12}$ J. P. Perdew, M. Ernzerhof, and K. Burke, J. Chem. Phys. 105, 9982 (1996).

${ }^{13}$ P. E. Blöchl, Phys. Rev. B 50, 17953 (1994).

${ }^{14}$ H. J. Monkhorst and J. D. Pack, Phys. Rev. B 13, 5188 (1976).

${ }^{15}$ C. Freysoldt, J. Neugebauer, and C. G. Van de Walle, Phys. Rev. Lett. 102, $16402(2009)$.

${ }^{16}$ G. Henkelman, B. P. Uberuaga, and H. Jonsson, J. Chem. Phys. 113, 9901 (2000).

${ }^{17}$ M. Bockstedte and M. Scheffler, Z. Phys. Chem. (Leipzig) 200, 195 (1997).

${ }^{18}$ D. Weiler and H. Mehrer, Philos. Mag. A 49, 309 (1984).

${ }^{19}$ J. A. Van Vechten, J. Phys. C 17, L933 (1984).

${ }^{20}$ F. El-Mellouhi and N. Mousseau, Phys. Rev. B 74, 205207 (2006).

${ }^{21}$ F. El-Mellouhi and N. Mousseau, Physica B 401-402, 658 (2007). 